\title{
A Comparison of Human Reliability Analysis Technique Using SMART Emergency Operating Guidelines
}

\author{
Eun Mee Heo', Seong Nam Byun ${ }^{2}$, Hong Joon Park ${ }^{3}$, Geun Ok Park \\ ${ }^{1,3}$ Department of Industrial Engineering, The University of Kyunghee, Yong-in, 446-701 \\ ${ }^{2}$ Department of Industrial \& Management, The University of Kyunghee, Yong-in, 446-701 \\ ${ }^{4}$ Korea Atomic Energy Research Institute (KAERI), Daejeon, 305-353
}

\author{
Corresponding Author \\ Seong Nam Byun \\ Department of Industrial \& Management \\ Engineering, The University of Kyunghee, \\ Yong-in, 446-701 \\ Mobile : +82-10-6339-7081 \\ Email : snbyun@khu.ac.kr
}

Received : November 11, 2013

Revised : December 05, 2013

Accepted: January 08, 2014
Copyright@2014 by Ergonomics Society of Korea. All right reserved.

(c) This is an open-access article distributed under the terms of the Creative Commons Attribution Non-Commercial License (http:// creativecommons.org/licenses/by-nc/3.0/), which permits unrestricted non-commercial use, distribution, and reproduction in any medium, provided the original work is properly cited.
Objective: The purpose of this study is to select the methodology for SMR HRA which has characteristics that are different from existing nuclear power plants and digitalbased plants.

Background: We must assure safety to preoccupy export of technology to developing countries or countries interested in nuclear application. And we can be an advanced country in nuclear technology by securing original technology in the field of SMR such as SMART.

Method: THERP, which is the most representative HRA methodology among all, and RARA, which is the latest HRA methodology. This study compared and evaluated THERP and RARA.

Results: As a result of applying THERP and RARA methodologies which are based on LOCA EOG task analysis result, this research concluded that RARA has higher personal errors than THERP.

Conclusion: This study needs validation for LOCA, emergency operations, normal and abnormal scenarios since HRA methodology was only focused on LOCA scenario.

Application: The results of this study can apply as base line data when designing MMIS, which is the main control room of SMART, and when building a simulator.

Keywords: Human reliability analysis, Probabilistic Safety Assessment, SMART

\section{Introduction}

There is currently a growing trend that digital technology applies to almost all the equipment in safety fields including nuclear power, aviation, railway and satellite, due to analogue technology regression and digital technology advance.

Analogue facilities are replaced with digital facilities in the existing nuclear power plants, let alone new nuclear power plants. The need for safety assessment in the digital system is emphasized, according to such a change and many relevant researches are underway (Tang, 1998; Naser, 2004; Ciesielski, 2004; Ngyyen, 2004; HSE, 1998).

In the SMR (Small and Medium Sized Reactors) domain like SMART (System- 
integrated Modular Advanced ReacTor), stable performance and safety need to be ensured to enter nuclear power technologyadvanced country rank and gain an opportunity to preempt technology export to developing countries or nuclear power utilization-interested countries by securing original technology. The purpose of this study is to select methodology suitable for SMR HRA through revision of HRA (Human Reliability Analysis) for SMR PSA (Probabilistic Safety Assessment) like SMART. Towards this end, this study compared THERP (Technique for Human Error Rate Prediction) (Swain and Guttmann, 1983), which was the most representative HRA methodology used in the industry, and recently released RARA (Railway Action Reliability Assessment) (RSSB, 2004, 2012).

Many countries including Korea conduct PSA in the digital system. The PSA technique is still at its infant stage, and thus, its clear-cut system and methodology have yet to be established, which becomes a very urgent pending global issue. PSA has been used as a key method to comprehensively assess safety of nuclear power plants, since WASH-1400 (NUREG/CR-1278, 1983) was published. PSA actually has been used in various fields such as meeting authorization and permission requirements, drawing system's optimum design drafts and easing regulations. As a result of PSA carried out for nuclear power plants thus far, 40 70\% of core damage frequency (CDF) has been identified to be related with human actions (Doughtery and Fragola, 1988; NEA, 2004). For this reason, it is very important to properly handle human actions in PSA. To enhance nuclear power plant's safety, errors caused by plant staff should be prevented or reduced. To this end, the analysis and evaluation on the errors need to be carried out in advance from the perspective of humans, the system users. HRA has been widely used in the nuclear power plant safety assessment field as a method to analyze and evaluate errors. Many limitations have been pointed out in the existing HRA, since it only focuses on quantitative assessment of error probabilities, and thus, methods stressing qualitative error analysis as an improved alternative have been recently developed (Dougherty, 1992; Hollnagel, 1997; USNRC, 1998).

The US NRC (2004) requires HRA to be considered from power the plant design stage. The NASA (2008) also specifies that HRA that affects system safety, as well as worker's safety, in designing all space systems, in which humans are involved, should be maximized. However, more diverse pending issues are drawn from the digital system's HRA under the situation that clear-cut system and methodology have yet to be established globally like digital system's PSA, as the speed of digital technology development becomes faster. For example, information quantity that can be viewed on a computer screen is limited, and thus the information should be displayed in sequence (Flach et al., 1995; Allen, 2007). This may cause continual accidents, because of a difficulty in effective coping with the information on the next page, if important information is ignored in the previous page (Bullemer et al., 2011).

SMR like SMART shows two different characteristics from the existing analogue-based control room tasks: First, SMR is designed with digital-based main control room like APR-1400, and thus the task is different from existing control room operation tasks. Second, SMR adopts compact computer-based indicator and controller so that two people can operate in normal operation unlike the composition of an operator from the existing main control room such as APR-1400. This study aims to select the methodology for SMR HRA that has characteristics different from the existing nuclear power plants and digital-based nuclear power plants. Figure 1 shows the research system for SMR HRA methodology selection.

\section{Consideration of HRA Existing Methodologies}

Although, human errors are confirmed to be major contributing factors to nuclear power plant risks through nuclear power plant accidents, operation experience and PSA, the accurate analysis of HRA is difficult, due to the lack of technological grounds, and the uncertainties of results have been recognized as high. Human error analysis system generally consists of mostly observable behaviors in HRA. In the nuclear power and aviation industries, various HRA methods have been developed and used for safety assessment. The typical methods include THERP, HEART (Human Error Assessment and Reduction Technique), ASEP (Accident Sequence Evaluation Program), which is the revised version of THERP, and HCR (Human Cognitive Reliability). The first generation 


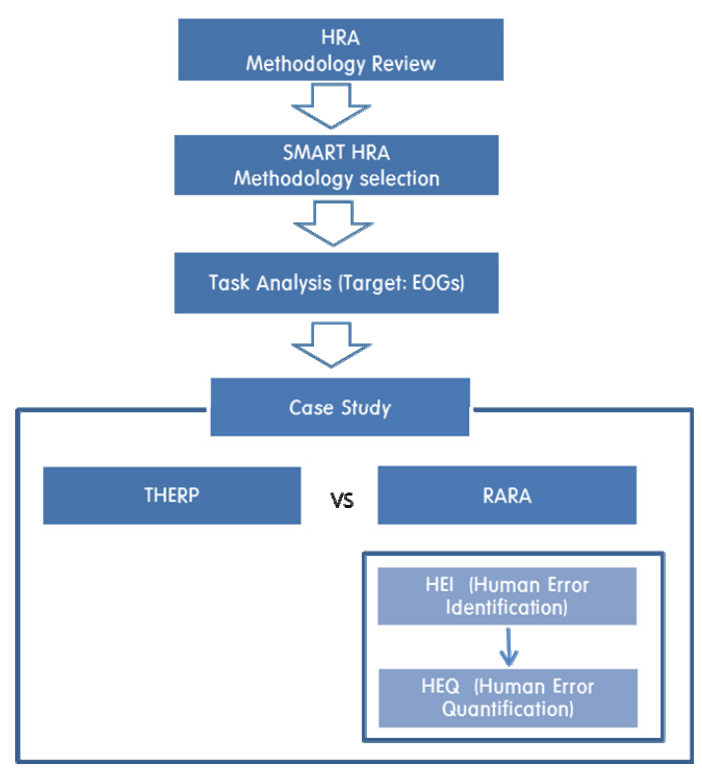

Figure 1. Structure of research

HRA methods including SLIM (Success Likelihood Index Method) mostly focuses on quantitative analysis from an engineering perspective, rather than analyzing the fundamental causes or structure of errors. They also did not properly handle errors caused in a series of decision making process determining behaviors to respond, after humans recognize problems and judge situation by evaluating focused on externally observable human tasks. As research results on human's cognitive characteristics and limitations are presented, due to the recent development of cognitive engineering and cognitive psychology, the problems on the existing HRA methods have been raised and movement to develop new methods have become active. Consequently, the following new methods have been proposed: CREAM (Cognitive Reliability and Error Analysis Method) and ATHEANA (A Technique for Human Error Analysis), the second generation; IDAC (Information Decision Action in crew Context), the third generation. With the advent of these methods, the existing HRA's typical limitation, a cognitive error analysis, has become possible at engineering level. The second and third generation methodologies, however, do not offer specific tools or guidelines, and therefore, they have a limitation of depending upon judgment based on analyst's experience and collected information. Even though there are various HRA methodologies, these techniques basically have been influenced by Rasmussen's (1981) skill based, rule based and knowledge based techniques, Reason's (1990) slips, lapses, mistakes and violations classification. Shorrock and Kirwan (2002) mentioned major problems including low usability (e.g. lack of structure, excessive requirements for supporting analysis, excessive jargon or excessive resolution), low contextual validity (particularly important for PSFs) and limited applicability (e.g. to skill based and rule-based performance only, to small-scale systems or applications only; to retrospective or predictive use only) on Rasmussen et al.'s (1981) HEI (Human Error Identification) technique or HRA techniques. HEI or HRA methodologies need to provide context and explanations on the realistic standards that accident investigators or designers can use or on the methodologies that can be easily and quickly used by them. HEI or HRA methodologies, however, are restricted to specific domains, depend on experiences too much and offer information that can explain errors just fragmentally. For error classification techniques to be usefully used, they need to provide standards with more diverse error selection scopes than the precious techniques. Various categories (standards) should be clearly classified and the details need to be understood easily (Shorrock and Kirwan, 2002). To select the actually applied methodology among various HRA methodologies, PSA reports, where HRA methodology was drawn up, were reviewed (Hanaman 1984; Electricite de France 1990; Institute de Protection et de Nucleaire 1990). Typical HRA methodologies, THERP and the THERP's revised version, ASEP, were confirmed to be used. In this context, this study evaluates whether the most representative HRA methodology, THERP, is suitable for SMR HRA. There is a need to identify the matters to consider in case of HRA analysis and necessary information to 
select SMR HRA by additionally reviewing the existing HRA processes and methods. This study selected five typical published methodologies, THERP, HEART, SPAR-H, ATHENANA and CREAM, except expert judgment, among various HRA methodologies, and then carried out status analyses on each methodology, and HEP (Human Error Probability) and PSF (Performance Shaping Factors). Of the five HRA methodologies, the rest except HEART and CREAM were classified into diagnosis error and operation error, and then HEP was defined. For operation errors, they were drawn by reflecting PSF items in basic HEP values. For the methodologies other than THERP, the experience data obtained by non-nuclear power fields in the 1960s and the THERP data built by expert judgment were used with basic HEP values. PSFs used in the typical five HRA methodologies have been summarized. For the used PSFs, 3 to 27 various numbers of PSFs are used. Of the PSFs used in the five methodologies, the PSFs commonly used or mentioned in numerous methodologies are as follows:

- Experience/Training

- Stress (Work load)

- MCR environment _ (THERP N/A)

- Time availability _ (THERP N/A)

- Complexity

- Procedure _ (THERP N/A)

RARA based on NARA (Nuclear Action Reliability Assessment) is the third generation HRA methodology that presents those common factors specifically. RARA is drawn up with specific items so that it can be matched with the HEQ (Human Error Quantification) PSFs of HEART, after drawn up with HEI PSFs primarily by classifying specific operation environmental factors as shown in Table 1. This study proposes a method suitable for SMR HRA, according to the comparative assessment of the most recent HRA method, RARA that supplements TRACEr (Technique for the Retrospective and predictive Analysis of Cognitive Errors), HEART and NARA assessment systems and a typical HRA methodology, THERP.

Table 1. Sample of RARA vs. HEART

\begin{tabular}{|c|c|}
\hline RARA PSF (HEI PSF) & HEQ PSFs to consider (HEART PSF) \\
\hline Motivation/Attitudes & \multirow{8}{*}{ Ability to detect and perceive } \\
\hline Emotional state (Anxiety/Panic/Anger/Depression) & \\
\hline Concentration & \\
\hline Confidence & \\
\hline Fatigue & \\
\hline Time stress & \\
\hline Load stress & \\
\hline Time Sharing & \\
\hline Duration of stress & Concentration \\
\hline Job satisfaction & \multirow{2}{*}{ Consistency of displays } \\
\hline Circadian rhythm & \\
\hline
\end{tabular}

\section{Task Analysis for SMART HRA Method Selection}

A task analysis needs to be conducted to undertake SMR HRA. This study applies HTA (Hierarchical Task Analysis) having merits 
that it is economical in collecting and comprising information and that can concentrate on sub-task analysis in addition to entire tasks among various task analysis techniques (Kirwan 1992). This study selected the task of LOCA (Loss Of Coolant Accident) from EOG (Emergency Operating Guidance), and the task analysis results are demonstrated in Figure 2. The upper tasks are 102 drawn up in LOCA EOG and they are divided into 5 stage sub-tasks maximum: 326 tasks to perform have been drawn as a result of entire tasks analysis.

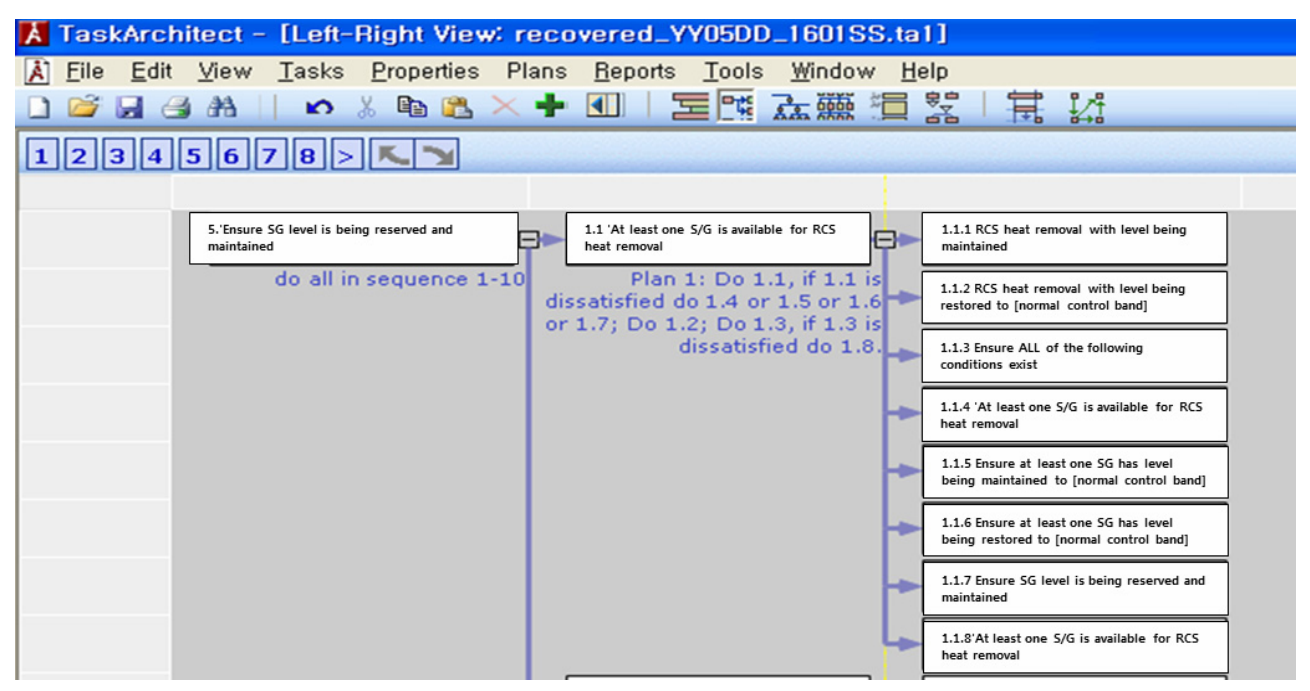

Figure 2. Result of task analysis

Based on 326 tasks analysis results, the following two tasks were selected to apply THERP and RARA methodologies. The reason is that operation can be undertaken through computer display, as the MCR environment changes to digital equipment.

- TASK A: 5.1 Selection of Safety Injection train A \& C (SIAC) directory

- TASK B: 5.2 Confirmation of SIAS warning

SMR has two characteristics different from the existing analogue-based control room task. For task analysis, this study first analyzed task stage by operators and task object. Necessary information was divided into information provision object (operator, display) and action object to which an action is taken (operator, control equipment). The analysis was conducted by classifying into behavior unit or information checking and collecting stage, situation identification and decision making stage and work performing stage. First, SMR conducts tasks through navigation that navigates desired display using computer device, instead of manipulating control like existing MCR. Second, monitoring of feedback, error recovery and correction, due to individual display unit operation, is important for the SMR operated by two operators in the case of normal operation.

\subsection{Case study}

HEI for quantitative analysis, based on task analysis results, is classified into THERP and RARA's GET (Generic Error Type) as shown in Table 2. THERP is analyzed as operation failure in terms of tasks $A$ and $B$ between diagnosis failure and operation failure. RARA is derived from perception failure and decision failure as errors that can select other directory in task A. RARA can be analyzed as detection failure, omission, perception failure and memory failure in task $B$. 
Table 2. Qualitative analysis

\begin{tabular}{|c|c|c|}
\hline TASK & THERP & RARA_GET \\
\hline \multirow{2}{*}{ A } & \multirow{2}{*}{ Error of commission } & Perception \\
\hline & & Decision \\
\hline \multirow{5}{*}{ B } & \multirow{5}{*}{ Error of commission } & Detection \\
\hline & & Omission \\
\hline & & Perception \\
\hline & & Memory \\
\hline & & Decision \\
\hline
\end{tabular}

\subsection{THERP Method}

THERP developed by Swain and Guttmann (1983) has been the most typically used HRA methodology since it was used by WASH-1400 (U.S.NRC.1997) for the first time. THERP is analyzed to be diagnosis failure and operation failure. The diagnosis failure is calculated by multiplying basic diagnosis error rate by correction value considering performance shaping factors (PSFs) through entering extra diagnosis time, so as to calculate final diagnosis error rate. The operation failure calculates entire operation errors by evaluating each unit work's error probability, after classifying the tasks concerned into several unit tasks.

Although, THERP classified many performance shaping factors (PSFs), it uses partial portion of them extremely including stress level, experience level and allowed time. THERP reflects the extent of influence on task performance by classifying stress into four levels: very low task load, optimum task load, heavy task load and threat stress. THERP takes into account five levels regarding dependence between tasks, namely, zero dependence (ZD), low dependence (LD), moderate dependence (MD), high dependence $(H D)$ and complete dependence (CD). The estimated probability value on each behavior is decided using the Databank (NUREG-1278) of THERP handbook. General assessment process is presented below:

- Calculate the probability of failure path by allocating HEP by task.

- Modify correction coefficient in consideration of stress, experience level and recovery factors.

- Calculate conditional probability in consideration of dependence between operators.

- Calculate final HEP.

Table 3 shows the results analyzed according to assessment process above. Tasks A and B of the diagnosis error and operation error are classified into operation error, because the tasks $A$ and $B$ are operating tasks of RO (Reactor Operator). In view of LOCA situation, the experience level is 5, according to THERP handbook's databank and is under the moderately high (MH) task load

Table 3. Quantitative analysis using THERP

\begin{tabular}{c|c|c|c|c|c|c|c|c|c}
\hline TASK & Failure & BHEP & Experience & Stress & Dependence & EF & HEP & $\begin{array}{c}\text { Recovery failure } \\
\text { probability }\end{array}$ & $\begin{array}{c}\text { Total } \\
\text { HEP }\end{array}$ \\
\hline A & $\begin{array}{c}\text { Error of } \\
\text { commission }\end{array}$ & 0.003 & 5 & MH & ZD & 5 & $0.003 \star 5=0.015$ & $\begin{array}{c}\text { SRO: } 0.508 \\
\text { TO: } 0.145\end{array}$ & 0.001105 \\
\hline B & $\begin{array}{c}\text { Error of } \\
\text { commission }\end{array}$ & 0.003 & 5 & MH & ZD & 5 & $0.003 * 5=0.015$ & $\begin{array}{c}\text { SRO: } 0.508 \\
\text { TO: } 0.145\end{array}$ & 0.001105 \\
\hline
\end{tabular}


situation. Due to the existence of task dependence between operators, $R O$ was assumed to be $Z D$, the dependence between $R O$ and SRO (Senior Reactor Operator) to be HD and the dependence between RO and TO (Turbine Operator) to be MD. BHEP on the task performance failure was 0.003 . If task dependence is $Z D$, the error factor (EF) becomes 5 . Because it was assumed that $R O$ error correction failed and dependence between the two operators was HD in SRO, the error probability by SRO becomes 0.508 . Because, it was assumed that correction of errors caused in performing RO tasks failed and dependence between the two operators became MD in TO, the error probability by TO becomes 0.145 . Total error probability becomes 0.001105 by multiplying the operation error value 0.015 that considered RO's stress level on task A and each operation error value of SRO and TO, namely, 0.508 and 0.145 , respectively. In task $B$, the same error values are drawn.

The tasks of nuclear power plants have a feature that normal and abnormal situations are clearly divided. THERP among the current methods presents the following with the table of THERP handbook: the change of estimated bounds derived from difference between operators, difference in analyses, uncertainty of modulization and uncertainty on actual HEP, namely, UCB (Uncertainty Bound) and difference between operators. This reflects total uncertainty bounds (TUB) on average estimates by demonstrating HEPs' distribution.

However, THERP is the first generation methodology and is aligned to nuclear power plant's task environment, but has a weakness in that THERP does not take into account operation characteristics of operator unit or workers' individual characteristics in analyzing task dependence.

\subsection{RARA Method}

RARA method consists of HEI stage, which is a qualitative analysis of human errors that referenced TRACEr (domain of air traffic control) for railway-unique HRA method by British RSSB and HEQ stage, which is the quantitative analysis referencing HEART and NARA. Through the flowchart format of human error check, consistent error check and classification are possible. In the case of RARA module, HEI and HEQ are systematically connected and examples are contained. All in all, the RARA method has an advantage that HRA analysis can be conducted easily. The HEI analysis process of RSSB-HRA is as follows:

- Preparation- data and information collection

- Identify the possible EEM (external error mode).

- Identify the possible cognitive Domains, IEM (internal error mode) and PEM (psychological error mechanism).

- Identify the PSFs (performance shaping factors) that could influence the occurrence of the error.

As a preliminary stage, task information required for error analysis is collected and error analysis is carried out, based on task analysis in the information collection stage. As for external error type, a total of 24 error types are presented from the domains of selection and quality, timing and sequence and communication. Cognitive domain classifies 5 domains - perception, decision, memory, action and violations, based on Wicken's human information processing model. Internal error type and psychological mechanism present manifestation-possible error types and induction mechanism, according to each cognitive domain. The cognitive domain identifies PSFs that affect error generation on each identified error.

HEQ process is similar to the analysis process of HEART, but HEART's generic task type (GTT) was changed to general error type (GET), and PSF system has been partially revised to be suitable for railway environment. Also, user guidelines on the influence assessment of the PSFs were added.

HEQ process is as follows: 
- Decide GEP(generic error probability) according to GET.

- Select task situation-related PSFs.

- Evaluate influences of the selected PSFs.

- Calculate final HEP.

GET classifies 7 error types by each cognitive stage, according to Wickens' human information processing model. For each error type, GEP, upper value, median value and lower value are presented. Final HEP can be calculated using the following formula that considers PSF weight (W(i)) and influence $(R(i))$ on GEP in the same way as the HEART system:

Final HEP $=$ GEP * II $[R(i) *(W(i)-1)+1]$

According to RARA analysis process, Table 4 shows HEI results analyzed with IEM and PEM according to EEM in terms of tasks A and B. Because Task A has a possibility to select another directory, EEM becomes the 'Right action on wrong object'. 'PerceptionMisidentification (visual)', 'Decision-Incorrect decision', 'Action-Selection error' are selected in IEM, according to cognitive domain, decision making domain and action domain with regard to EEM. PEM becomes 'Memory-Mis-learning' and 'Decision-Incorrect knowledge', according to memory domain and psychological error mechanism on the internal error type decided above.

Based on the qualitative analysis in Table 4, GET is selected and the values concerned are allocated to allocate GEP for quantitative analysis. GET for 'Right action on wrong object' of task $A$ is perception and decision, and each GEP has nominal values, 0.00002 and 0.00004 , respectively. When it comes to calculation of the concerned human error's final HEP in line with the formula by multiplying

Table 4. Qualitative analysis using RARA

\begin{tabular}{|c|c|c|c|}
\hline TASK & EEM & IEM & PEM \\
\hline A & $\begin{array}{l}\text { Right action on } \\
\text { wrong object }\end{array}$ & $\begin{array}{l}\text { Perception-Misidentification(visual) } \\
\text { Decision-Incorrect decision } \\
\text { Action-Selection error }\end{array}$ & $\begin{array}{l}\text { Memory-Mis-learning } \\
\text { Decision-Incorrect knowledge } \\
\text { Decision-Lack of knowledge } \\
\text { Perception-Perceptual tunnelling } \\
\text { Action-Thoughts leading to action } \\
\text { Perception-Stimulus overload } \\
\text { Perception-Spatial confusion } \\
\text { Action-Spatial confusion } \\
\text { Perception-Perceptual confusion } \\
\text { Memory-Similarity interference }\end{array}$ \\
\hline \multirow{5}{*}{ B } & Action omitted & \multirow{5}{*}{$\begin{array}{l}\text { Action-Incorrect information transmitted } \\
\text { Decision-Incorrect decision } \\
\text { Memory-Misrecall stored information } \\
\text { Memory-Prospective memory failure } \\
\text { Perception-Misidentification (visual) } \\
\text { Perception-Misread } \\
\text { Perception-No detection (visual) } \\
\text { Perception-Visual misperception } \\
\text { Violation-Routine violation } \\
\text { Violation-Situational violation }\end{array}$} & \multirow{5}{*}{$\begin{array}{l}\text { Action-Habit intrusion } \\
\text { Action-Spatial confusion } \\
\text { Decision-Incorrect knowledge } \\
\text { Decision-Misunderstanding } \\
\text { communication } \\
\text { Memory-Mis-learning } \\
\text { Memory-Similarity interference } \\
\text { Perception-Distraction/Preoccupation } \\
\text { Perception-Perceptual confusion } \\
\text { Perception-Perceptual discrimination failure } \\
\text { Perception-Perceptual tunnelling } \\
\text { Perception-Spatial confusion } \\
\text { Perception-Stimulus overload }\end{array}$} \\
\hline & $\begin{array}{l}\text { Unclear information } \\
\text { transmitted }\end{array}$ & & \\
\hline & $\begin{array}{l}\text { Incorrect information } \\
\text { transmitted }\end{array}$ & & \\
\hline & $\begin{array}{l}\text { Incomplete information } \\
\text { transmitted }\end{array}$ & & \\
\hline & $\begin{array}{l}\text { Right action on } \\
\text { wrong object }\end{array}$ & & \\
\hline
\end{tabular}


Table 5. Quantitative analysis using RARA

\begin{tabular}{|c|c|c|c|c|c|c|c|c|c|c|}
\hline TASK & EEM & GET & GEP & PSF & Multiplier & Effect & $\begin{array}{l}\text { Multiplier } \\
\text { * Effect }\end{array}$ & HER & HEP & $\begin{array}{l}\text { Total } \\
\text { HEP }\end{array}$ \\
\hline \multirow{5}{*}{ A } & \multirow{5}{*}{$\begin{array}{l}\text { Right action on } \\
\text { wrong object }\end{array}$} & Perception & 0.000044 & \multirow{5}{*}{$\begin{array}{l}\text { Operator } \\
\text { experience } \\
\text { Time availability } \\
\text { High workload } \\
\text { Positioning } \\
\text { and layout } \\
\text { Education and } \\
\text { training }\end{array}$} & 4.8 & 0.026 & 1.0988 & 0.00011499 & 0.0000019 & \multirow{5}{*}{0.005705} \\
\hline & & Response & 0.131 & & 11 & 0.045 & 1.45 & 0.34235652 & 0.0056998 & \\
\hline & & Decision & 0.000064 & & 6 & 0.086 & 1.43 & 0.00016726 & 0.0000028 & \\
\hline & & & & & 5 & 0.026 & 1.104 & & & \\
\hline & & & & & 2.3 & 0.03 & 1.039 & & & \\
\hline \multirow{18}{*}{ B } & \multirow{6}{*}{ Action omitted } & \multirow{3}{*}{ Detection } & \multirow{3}{*}{0.00017} & \multirow{6}{*}{$\begin{array}{l}\text { High workload } \\
\text { Education and } \\
\text { training } \\
\text { Information } \\
\text { quality \& } \\
\text { availability } \\
\text { Time availability } \\
\text { Risk perception } \\
\text { Positioning and } \\
\text { layout }\end{array}$} & 6 & 0.086 & 1.43 & \multirow{3}{*}{0.00053276} & \multirow{3}{*}{0.000008} & \multirow{18}{*}{0.003703} \\
\hline & & & & & 2.3 & 0.03 & 1.039 & & & \\
\hline & & & & & 4.9 & 0.057 & 1.2223 & & & \\
\hline & & \multirow{3}{*}{ Omission } & \multirow{3}{*}{0.00011} & & 11 & 0.045 & 1.451 & \multirow{3}{*}{0.00034473} & \multirow{3}{*}{0.0000057} & \\
\hline & & & & & 4 & 0.026 & 1.078 & & & \\
\hline & & & & & 5 & 0.026 & 1.104 & & & \\
\hline & $\begin{array}{l}\text { Unclear } \\
\text { information } \\
\text { transmitted }\end{array}$ & Interpretation & 0.0265 & $\begin{array}{l}\text { Information } \\
\text { quality \& } \\
\text { availability }\end{array}$ & 4.9 & 0.057 & 1.2223 & 0.03258530 & 0.0005425 & \\
\hline & $\begin{array}{l}\text { Incorrect } \\
\text { information } \\
\text { transmitted }\end{array}$ & Interpretation & 0.0265 & $\begin{array}{l}\text { Consistency of } \\
\text { displays }\end{array}$ & 1.2 & 0.03 & 1.006 & 0.03258530 & 0.0005425 & \\
\hline & $\begin{array}{l}\text { Incorrect } \\
\text { information } \\
\text { transmitted }\end{array}$ & Interpretation & 0.0265 & $\begin{array}{l}\text { Consistency of } \\
\text { displays }\end{array}$ & 1.2 & 0.03 & 1.006 & 0.04746899 & \multirow[t]{2}{*}{0.0007903} & \\
\hline & \multirow{4}{*}{$\begin{array}{l}\text { Right action on } \\
\text { wrong object }\end{array}$} & \multirow{4}{*}{ Interpretation } & \multirow{4}{*}{0.0265} & $\begin{array}{l}\text { Education and } \\
\text { training }\end{array}$ & 2.3 & 0.03 & 1.039 & 0.04746899 & & \\
\hline & & & & $\begin{array}{l}\text { Information } \\
\text { quality } \& \\
\text { availability }\end{array}$ & 4.9 & 0.057 & 1.2223 & & 0.0007903 & \\
\hline & & & & $\begin{array}{l}\text { Positioning and } \\
\text { layout }\end{array}$ & 5 & 0.026 & 1.104 & & & \\
\hline & & & & $\begin{array}{l}\text { Ability to detect } \\
\text { and perceive }\end{array}$ & 10 & 0.03 & 1.27 & & & \\
\hline & \multirow{5}{*}{$\begin{array}{l}\text { Right action on } \\
\text { wrong object }\end{array}$} & Interpretation & 0.0265 & \multirow{5}{*}{$\begin{array}{l}\text { Consistency of } \\
\text { displays } \\
\text { Positioning and } \\
\text { layout } \\
\text { Ability to detect } \\
\text { and perceive } \\
\text { Operator } \\
\text { experience } \\
\text { High workload }\end{array}$} & 1.2 & 0.03 & 1.006 & 0.05873153 & 0.0009778 & \\
\hline & & Memory & 0.00118 & & 5 & 0.026 & 1.104 & 0.00261522 & 0.0000435 & \\
\hline & & Decision & 0.000064 & & 10 & 0.03 & 1.27 & 0.00014184 & 0.0000023 & \\
\hline & & & & & 4.8 & 0.026 & 1.0988 & & & \\
\hline & & & & & 6 & 0.086 & 1.43 & & & \\
\hline
\end{tabular}


the drawn PSFs influence and weight, it is demonstrated as shown in Table 5.

The RARA classification system as shown in Tables 4 and 5 can be divided into error type and PSF system. For error type, the error type in the railway task environment is added and revised to generic psychological classification system based on human information processing model. However, this research was modified based on the error analysis in the aviation and nuclear power field tasks on the basis of TRACEr, HEART and NARA. For PSFs, the generic PSF classification system presented in the HEART is used. Therefore, the limitation that SMR HRA was applied to the railway industry is forecast to be overcome in terms of SMR HRA methodology selection. However, the selection of PSFs on the given task and environment depends on an analyst, and thus, there is a limitation that non-consistent selection and assessment may arise between analysts. The two modules of RARA, HEI and HEQ can be used independently, but the link is meager.

Since HEI, classified into EEM, IEM, PEM and PSF, is linked with HEQ there is a demerit that EEM, IEM and PEM are contracted to GET again. Unlike other methodologies, however, RARA's analysis process is systematized and proceduralized, and thus, there is an advantage that even beginners can smoothly apply it.

\section{Result}

Figure 3 shows the HRA quantification assessment results of the THERP and RARA methodologies on tasks A and B. THERP was classified as the same error, and the same HEP of 0.001105 was drawn. RARA was classified into different errors, according to cognitive error and psychological error mechanisms, and thus, 0.005705 and 0.003703 were drawn, respectively. Looking at tasks $A$ and $B$ alone, it is understood that RARA presents higher values than THERP.

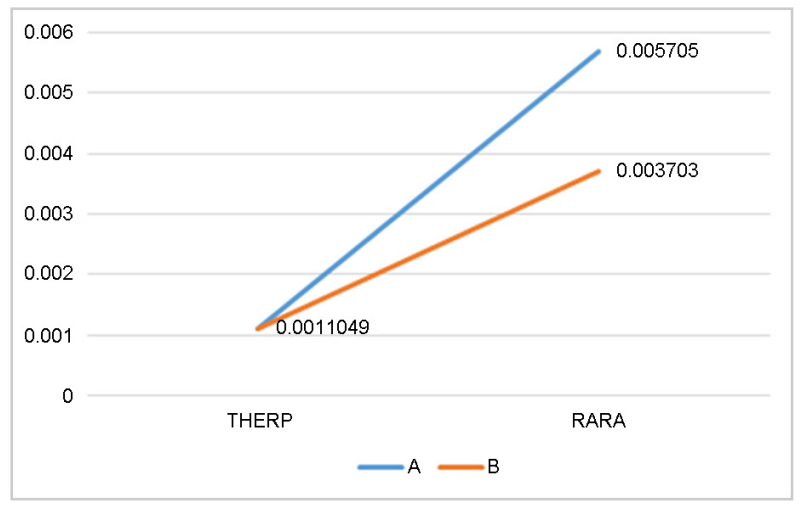

Figure 3. Comparison of tasks

As shown in the examples of tasks $A$ and $B$, the analytical results by applying the THERP and RARA methodologies, based on LOCA EOG task results, are demonstrated in Figure 4. For LOCA EOG, it can also be confirmed that RARA is higher human error probability than THERP. As a result of analyzing entire LOCA scenarios, RARA's detailed human error type can be identified, compared to THERP, and HEP value can be drawn by each human error type. This is ascribable to a decomposition approach that assesses error possibility by segmenting HEI as presented in RARA. 


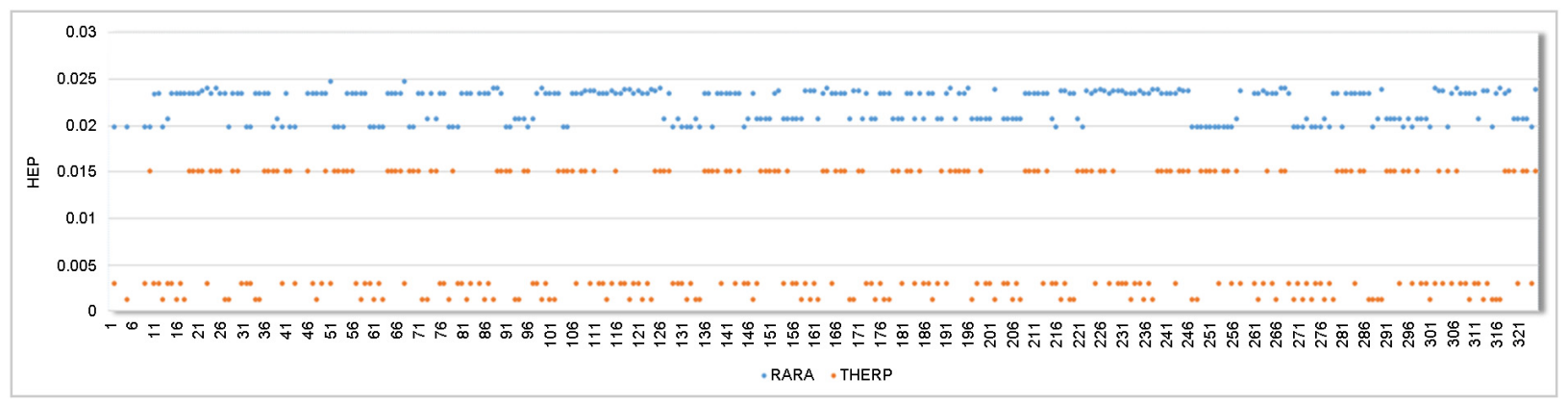

Figure 4. Comparison HRA methods

\section{Discussion/Conclusion}

In the nuclear power industry that carries out HRA in reality still the most widely use THERP that assesses by classifying performance procedures into certain unit work. THERP, however, is the first generation HRA methodology and is specialized in the analoguemode operation environment. DB also indicates human error types and HEP targeting the experience data and expert judgment obtained from the non-nuclear power fields in the 1960s. For this reason, the digitalized SMR human error type and probability cannot be forecast and there are no PSFs reflecting the digitalized MCR environment. Because, the nuclear power industry especially applies a conservative approach in terms of safety, it can be appropriate to apply RARA to SMR HRA, viewing from HEP values drawn from the LOCA scenario alone as shown in Figure 4.

This study decides that RARA, which offers detailed human error list to find human error classification and mechanisms in the digital environment and is based on TRACEr developed in the aviation industry and NARA developed in the nuclear power industry, is applicable to the SMR HRA method, although RARA is a methodology applied to the railway industry. The reason is that there is a need to check operators' cognitive errors and psychological effort mechanisms, according to shift into the digital environment and two operator's operation. Like the first generation HRA methodologies such as THERP and HEART, RARA has a limitation that has been applied to the analogue system, but, it provides detailed analyses on cognitive errors and psychological error mechanisms. Control-Display relationships and the structure of information and team relations, which are PSFs suitable for SMR operated by two operators in the digital environment, are reflected in RARA. RARA is expected to offer very reliable HRA, if it is used for SMR HRA, because RARA has modulized analysis methods and examples that can maintain objectivity maximum. This study, however, targeted only specific tasks of LOCA scenario, and thus, cannot be viewed as representing the entire digital environment on the operator's entire tasks. In this context, additional analyses on abnormal operation including LOCA, and normal and abnormal scenarios are needed. Besides, additional study on the PSFs of the digital environment and DB development through experiments using a simulator should be carried out.

\section{Acknowledgements}

This work was supported by the National Research Foundation of Korea (NRF) grant funded by the Korea government (MEST) (No. 2013M2B9A1025900).

\section{References}

ABB-CE, "System 80+ PRA", 1993. 
Cha, W.C., "A Comparative Study of Digital and Analogue System Environment to Retrieve Human Factor Elements for the Design of Operator Console, Journal of the Society of Korea Industrial and Systems Engineering, Vol.32, No.2, pp.140-146, June 2009.

Dave Ciesielski, Phil Liddle, "Qualified Software Tools For Safety I\&C Applications" NPIC\&HMIT 2004, Columbus, USA, 2004.

Dougherty, E., Human Reliability Analysis - Where should sthou turn? Journal of Reliability Engineering and System Safety 29 , 283-299, 1992.

Doughtery, E.M. and Fragola, J.R., "Human Reliability Analysis: A System Engineering Approach With Nuclear Power Plant Applications", SAIC, John Wiley \& Sons, 1988.

Eletricite de France, Probabilistic Safety Assessment of Reactor Unit 3 in the Paluel Nuclear Power Centre (1300 we), 1990.

Embrey, D., Humphreys, P., Rosa, E., Kirwan, B. and Rea, K., SLMMMAUD; An Approach to Assessing Human Error Probability Using Structured Expert Judgment, NUREG/CR-3518, Vol. I\&II 1984.

Gertman, D., Blackman, H., Marble, J., Byers, J. and Smith, C., "The SPAR-H Human Reliability Analysis Method", NUREG/CR-6883, 2005. USNRC.

Hannaman, G.W., Sprgin, A.J. and Lukic, Y.D., "Human Cognitive Reliability (HCR) model for PRA analysis", Drafts report, NUS-4531, EPRI Project RP 2170-3. 1984.

Health and Safety, Executive, Review of human reliability assessment methods, 2009.

Health and Safety Executive, The use of computers in Safety-Critical Applications, London, HSE books, 1998.

Hollnagel 1997, Hollnagel, E., CREAM - Cognitive reliability and error analysis method, Elsevier Science Publishers, 1998.

Hollnagel, E., CREAM - Cognitive reliability and error analysis method, Elsevier Science Publishers, 1998.

Institute de Protection et de Nucleaire, "A Probabilistic Safety Assessment of the Standard French 900 Mwe Pressurised Water Reactor", 1990.

Isaac, A., Shorrock, S.T. and Kirwan, B., Human error in European air traffic management: the HERA project. Reliability Engineering and System Safety, 75(2), 257-272, 2002.

Joseph Naser, "Generic Pre-Qualification of Digital Platforms for Safety Applications: A Success Story for Instrumentation and Control Modernization in Nuclear Power Plants", NPIC\&HMIT 2004, Columbus, USA, 2004.

Kaufuman 1999 Lori M. Kaufuman, barry W. Johnson, "Embedded Digital System Reliability and Safety Analysis" NUREG/GR-0020 USNRC 1999.

Kirwan, B., Plant control diagnostic failure - just a matter of time? In Proceedings of the IEEE Conference on Human Factors and Power Plants, Monterey, USA, June, pp.51-69, 1992a. 
Kirwan, B. (1992b), Human error identification in human reliability assessment. Part 2: detailed comparison of techniques, Applied Ergonomics 23(6), p.371, 1992.

Lee, J.H. and Bae, Y.N., "A study on the effect of digital and analogue control panels to user interface" Korea Design Science Research, Vol.4 No.2 (2001. 7) pp.95-104 1229-1072.

NASA (2008) Human-Rating Requirements for Space Systems, NASA Procedural Requirements, NPR 8705.2B, 2008.

NRC (2004) USNRC, Human Factors Engineering Program Review Model, NUREG-0711, Rev.2, US NRC, 2004

Nuclear Energy Agency, "Nuclear Regulatory Challenges Related to Human Performance" NEA No.5334, 2004.

Rail Safety and Standard Board (RSSB). Rail-specific HRA technique for driving tasks, Final report. 2004.

Rail Safety and Standard Board (RSSB), Railway Action Reliability Assessment user manual, 2012.

Rasmussen, J., Pedersen, O.M., Carnino, A., Griffon, M., Mancini, C. and Gagnolet, P., Classification System for Reporting Events Involving Human Malfunctions, RISO-M-2240, DK- 4000, Riso National Laboratories, Roskilde, Denmark, 1981.

Reason's, and Reason, J.T., Human Error. Cambridge, UK: Cambridge University Press, 1990.

Shorrock 2002a, The two-fold path to human error analysis: TRACEr lite retrospection and prediction, Safety Systems (Newsletter of the Safety-Critical Systems Club), 11(3), September 2002.

Shorrock 2002b, Error Classification for Safety Management: Finding the Right Approach, In Workshop on the Investigation and Reporting of Incidents 2002.

Swain, A.D. 1987, "Accident sequence evaluation program human reliability analysis procedure", NUREG/CR- 4772, USNRC. 1987.

Tang, D., Hecht, M., An, X. and Brill, R., "MEADEP and its application in dependability analysis for Nuclear Power Plants safety system", IEEE Tr. on Nuclear Science, Vol., 45 No.3, p.1014-1021, 1998.

Thunem, 2004 Atoosa Thunem, P.J., "Computerized Systems' Availability, Safety and Research at the OECD Halden Reactor Project", NPIC\&HMIT 2004, Columbus, USA, 2004.

Thuy Ngyyen, Dave Blanchard, Robert Flink, Glenn Lang, Ray Torok, Simplified Risk-Informed Assessment of Defence-in-Depth and Diversity for Digital I\&C Upgrades in Nuclear Power Plants, NPIC \& HMIT 2004, Columbus, USA, 2004.

USNRC. 1983 Swain, A.D., Guttmann, H.E., "Handbook of human reliability analysis with emphasis on nuclear power plant applications", NUREG/CR-1278, USNRC. 1983.

USNRC 1998 Regulatory Guide 1.174, "An Approach for Using Probabilistic Risk Assessment in Risk-Informed Decisions on Plant-Specific Changes to the Licensing Basis" July 1998.

USNRC, Technical basis and implementation guidelines for a technique for human event analysis (ATHEANA), NUREG-1624, Rev.1, 
US NRC, 2000.

WASH-1400 "Reactor safety study -An Assessment of accident risks in U.S. Commercial Nuclear Power Plant" NUREG-75/014 Wash-1400, 1975.

Whitehead, D. et al., "Evaluation of Potential Severe Accident During Low Power and Shutdown Operations at Grand Gulf Unit I" NUREG/CR-6143 S.N.L, June 1994.

Williams, J.C., "A data-based method for assessing and reducing human error to improve operational performance", Proceedings of the IEEE Fourth Conference on Human Factors and Power Plants, Monterey, California. 1988.

\section{Author listings}

Eun Mee Heo: hem2040@khu.ac.kr

Highest degree: MS, Department of International Management, Kyunghee University

Position title: Ph.D. candidate, Department of Industrial Engineering, Kyunghee University of Yong-in

Areas of interest: Human Factors in Nuclear Power Plant, HRA, HCI, Team Performance

Seong Nam Byun: snbyun@khu.ac.kr

Highest degree: Ph.D. Department of Industrial Engineering, The University of Michigan

Position title: Professor, Department of Industrial \& Management Engineering, Kyunghee University of Yong-in

Areas of interest: Human Factors in Nuclear Power Plant, Training, HCI, HRA

Hong Joon Park: groovyhong@khu.ac.kr

Highest degree: MS, Department of Industrial Engineering, Kyunghee University

Position title: Ph.D. candidate, Department of Industrial Engineering, Kyunghee University

Areas of interest: Human Factors in Nuclear Power Plant, Training, HCI

Geun Ok Park: gopark@kaeri.re.kr

Highest degree: Ph.D. Department of Computer Engineering, Kongju National University

Position title: Project Manager, Department of Research Reactor Engineering, Korea Atomic Energy Research Institute

Areas of interest: Human Factors in Nuclear Power Plant, Training System in Nuclear Industry, Simulator, HMI 